\title{
Synthesis of Schiff's Bases from 2-Amino-4H-1, 3-Oxazine / Thiazine and Substituted Aldehydes and their Transition Metal Complexes
}

\author{
D.T. Biradar ${ }^{1}$, Dr. M. B. Swami ${ }^{2}$ \\ ${ }^{1}$ Department of Chemistry, Research Centre, Shivaji Mahavidyalaya, Udgir, Dist.Latur \\ ${ }^{2}$ Dept. of Chemistry, Bahirji Smarak Mahavidyalaya, Basmath, dist. Hingoli-431512[M.S]
}

\begin{abstract}
Objective: Many methods can be found in literature for the synthesis a Oxazines and Thiazines. Few are reported for one-pot multi component cyclo-condensation of an alkayane urea / Thiourea and Aldehyde to 2- amino-4H-1, 3-Oxazines or Thiazines. Different catalysts are reported like Trifluoro-acetic acid, glacial acetic acid under reflux condition Mandal and Co-workers have utilized perchloric acid $\left(\mathrm{HClO}_{4}\right)$ supported on silica gel as catalyst under solvent free condition ytterbium trifluorate $\left[\mathrm{Yb}(\mathrm{OTF})_{3}\right]$ plays role of Lewis acid. It is also used in several Diel's-Alder cyclo-addition reactions.

Inspired these observations, development of simple effective synthetic strategy for the synthesis of 2 -amino- $4 \mathrm{H}-1,3-$ Oxazium \& Thiazines was attempted.

Material and Methods: One pot multi-component synthesis of Schiff's base as new ligand an their complexes with Ni, $\mathrm{Fe}, \mathrm{Co}$ and $\mathrm{Cu}$ and developed using 2-amino-4H-1,3 Oxazines /Thiazines and substituted Aldehydes, different catalyst are used to synthesize of 2-amino 4H-1,3 Oxazines. Its characterization is confirmed by taking IR, NMR spectra.

The Schiff's base act as ligand is bidentate, when it is treated to metal complexes in 1:1 ratio (metal: ligand) complexes are formed. Result: By using the bioactive molecules like 2-amino-4H-1, 3 Oxazine / Thiazines, Schiff's Bases can be synthesized by using different aldehydes. These Schiff's Bases has antitumor, antipyretic, anti-inflammatory property. And these Schiff's bases treated with bioactive metals like $\mathrm{Co}, \mathrm{Fe}, \mathrm{Cu}, \mathrm{Zn}$ which enhances their bio-activity.
\end{abstract}

Conclusion: All these synthesized Schiff' Bases and their metal complexes having bioactive properties.

KEYWORDS: Coordination complexes and Characterization, Oxazines and substituted aldehydes, Synthesis of Schiff's bases, 2amine-4H-1, 3 .

\section{INTRODUCTION}

In chemical research and industry has increased efficient, economical clean short time procedure increases attention in recent years ${ }^{\mathbf{1}}$. Development of such methods is great demand in coordination chemistry.

Compounds containing an Oxazine or Thaizine moiety have been found to passes a wide range of bioactivities like antifungal antibiotic ${ }^{2-3}$. A derivative of oxazine has also exhibited anti- HIV activity

Many methods can be found in literature for the synthesis a oxazines and thiazines. Few are reported for one-pot multicomponent cyclocondensation of an alkayane urea/thiourea and aldehyde to 2- amino-4H-1, 3-oxazine or thiazine ${ }^{3}$. Different catalysts are reported like trifluoraoacetic acid glacial acetic acid under reflux condition Mandal and co-workers have utilized perchloric acid $\left(\mathrm{HClO}_{4}\right)$ supported on silica gel as catalyst under solvent free condition ytterbium trifluorate $\left[\mathrm{yb}(\mathrm{OTF})_{3}\right]$ plays role of Lewis acid It is also used in several Diel's-Alder cyclo-addition reactions ${ }^{4}$.

Inspired these observations, development of simple effective synthetic strategy for the synthesis of 2-amino-4H-1, 3Oxazine \& Thiazine was attempted ${ }^{5-7}$.

Schiff's bases also have applications of ant-inflammatory, antitumor, antibiotic, antifungal activities. We want to enhance the bioactivity by containing these two moiety using different methods are used ${ }^{8-9}$. To synthesize the Schiff's bases. Different methods are reported like constant stirring at moderate temperature use of catalysts like $\mathrm{H}_{2} \mathrm{SO}_{4}, \mathrm{AlCl}_{3}$ etc.

If such bases are treated with biologically active metals like $\mathrm{Zn}, \mathrm{CO}, \mathrm{Cu}, \mathrm{Fe}$ it enhance the biological activity of compounds. 


\section{International Journal of Current Science Research and Review}

ISSN: 2581-8341

Volume 04 Issue 07 July 2021

DOI: 10.47191/ijcsrr/V4-i7-09, Impact Factor: 5.825

IJCSRR@ 2021

www.ijcsrr.org

\section{Preparation of 2-Amino-4H-1, 3-Oxazine / Thiazine:}

A solution of benzaldehyde ( $1 \mathrm{~m}$ mole), (2- bromovinyl) $1 \mathrm{~m}$ mole and Urea $3 \mathrm{~m}$ mole in water ( $3 \mathrm{~m}$ mole) was taken in $20 \mathrm{ml}$ vial and subject to M.W. irradiated at $\left(120^{\circ}-250^{\circ} \mathrm{C}\right)$ reaction completed monitored by TLC, reaction mixture was cooled, filter wash with acetone and re-crystallize by ethanol to give pure product.<smiles>[R]c1ccccc1C=O</smiles><smiles>[X]C(=N)N</smiles>

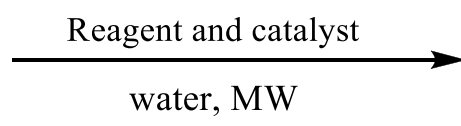<smiles>[R][X]1c([R])cc(-c2ccccc2)nc1N</smiles>

Table. No.1

\begin{tabular}{|c|c|c|c|c|}
\hline Sr. NO. & $\mathrm{R}_{1}$ & $\mathrm{R}_{2}$ & $\mathrm{X}$ & Yield in \% \\
\hline 1 & $\mathrm{H}$ & $\mathrm{C}$ & $\mathrm{O}$ & 89 \\
\hline 2 & $\mathrm{P}^{-\mathrm{CH}_{3}}$ & $\mathrm{C}_{6} \mathrm{H}_{5}$ & $\mathrm{O}$ & 72 \\
\hline 3 & $\mathrm{O}-\mathrm{OCH}_{3}$ & $\mathrm{C}_{6} \mathrm{H}_{5}$ & $\mathrm{O}$ & 84 \\
\hline 4 & ${\mathrm{M}-\mathrm{NO}_{2}}$ & $\mathrm{C}_{6} \mathrm{H}_{5}$ & $\mathrm{O}$ & 85 \\
\hline 5 & $\mathrm{H}$ & $\mathrm{C}_{6} \mathrm{H}_{5}$ & $\mathrm{~S}$ & 76 \\
\hline 6 & $\mathrm{P}-\mathrm{CH}_{3}$ & $\mathrm{C}_{6} \mathrm{H}_{5}$ & $\mathrm{~S}$ & 72 \\
\hline 7 & $\mathrm{P}-\mathrm{Cl}$ & $\mathrm{C}_{6} \mathrm{H}_{5}$ & $\mathrm{~S}$ & 77 \\
\hline 8 & $\mathrm{P}-\mathrm{NO}_{2}$ & $\mathrm{C}_{6} \mathrm{H}_{5}$ & $\mathrm{~S}$ & 66 \\
\hline
\end{tabular}

Preparation of Schiff's bases :

Take $\mathrm{m}$ mol of 2- amino 4H-1, 3 Oxazine or Thiazine compound in mortar and add benzaldehyd add 2-3 drops of ethanol grind for 10-15 min or it stir for 1-2 Hrs. We get the products. Pour this product on crushed ice, filter and re-crystallize it using ethanol.

Strong singlet at 9.41 in NMR and 3400-3500 and in IR does no observed indicates $\mathrm{NH}_{2}$ convert in $\mathrm{R}_{2} \mathrm{C}=\mathrm{NR}$ (imines) Band at 3650-3584 due to free -OH band at 1689-1471 due to imines stretching<smiles>[R][X]1c([R])cc(-c2ccccc2)nc1N</smiles><smiles>[R]Cc1cc(-c2ccccc2)nc(/N=C/c2ccccc2O)[X]1[R]</smiles> 
ISSN: 2581-8341

Volume 04 Issue 07 July 2021

DOI: 10.47191/ijcsrr/V4-i7-09, Impact Factor: 5.825

\section{Synthesis of Metal complexes:}

Solution of ligand in methanol \& metal salt were mixed thoroughly in 1:1 and 2:1 metal legend ratio and $1 \%$ mixed $\mathrm{KOH}$ solution in methanol was added to adjust PH between 7-8 and reflux for 1-2 hr. We get complex which monitored on TLC. $\mathrm{M}\left(\mathrm{CH}_{3} \mathrm{COO}\right)_{2}\left(\mathrm{H}_{2} \mathrm{O}\right)_{4}+1$ or $2 \mathrm{Ln} \square \mathrm{M} \ln$ or ${\mathrm{M} \mathrm{ln}_{2}}$ $\mathrm{M}=\mathrm{Cu}, \mathrm{Fe}, \mathrm{Co}, \mathrm{Zn}$

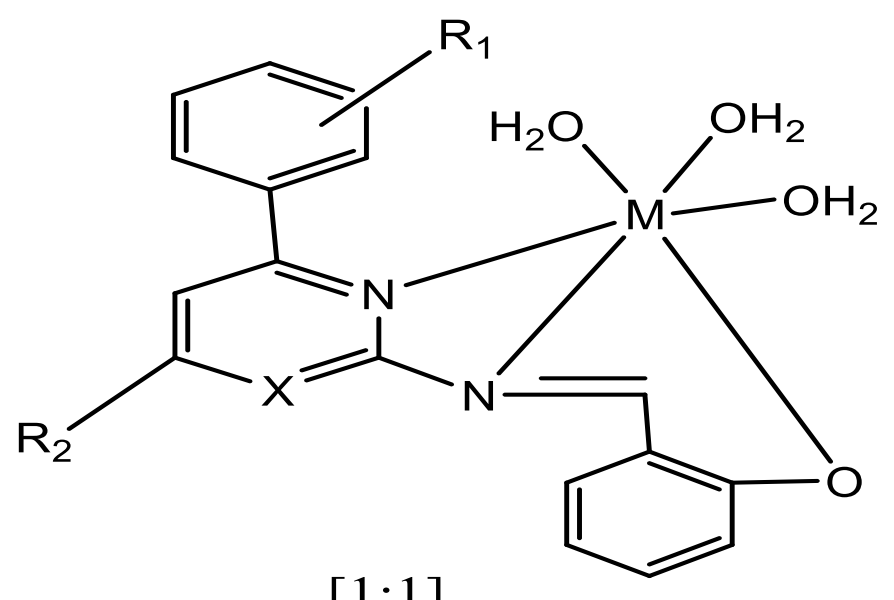

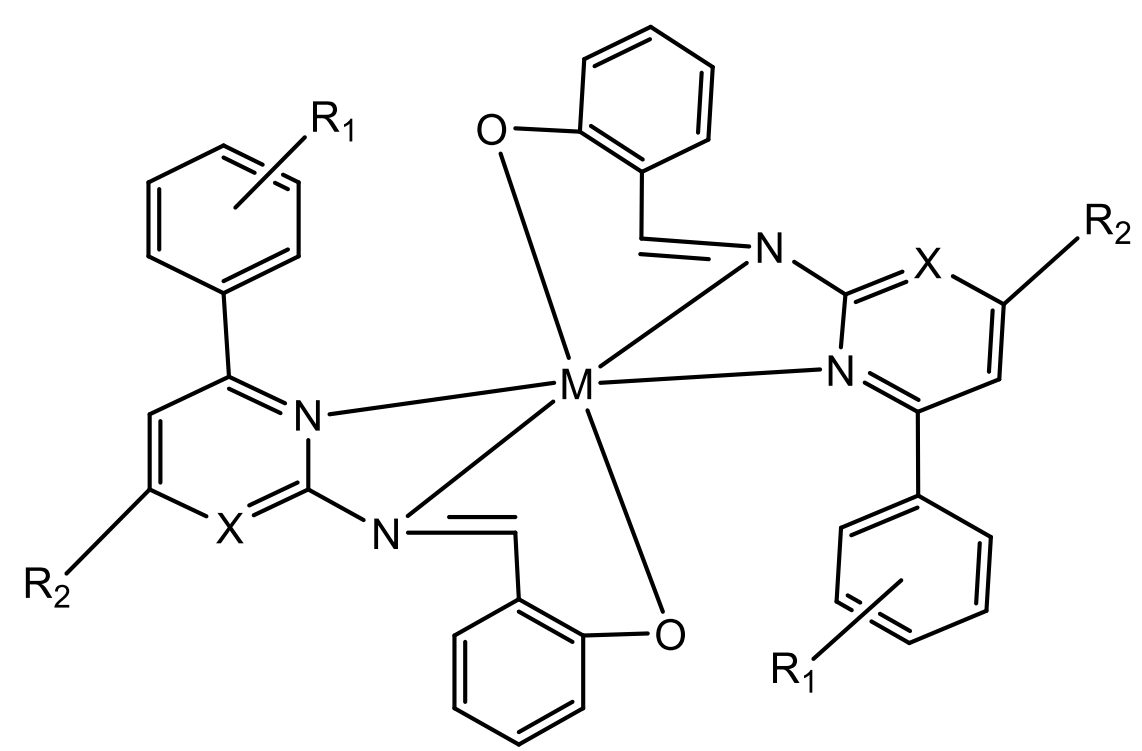

Select spectroscopic data Band at 525-425,450-350 NMR- H Ar. at7.60

\section{RESULT AND DISCUSSION}

By using the bioactive molecules like 2-amino-4H-1, 3 Oxazine / Thiazines, Schiff's Bases can be synthesized by using different substituted aldehydes. These Schiff's Bases has antitumor, antipyretic, anti-inflammatory property.

And these Schiff's bases treated with bioactive metals like $\mathrm{Co}, \mathrm{Fe}, \mathrm{Cu}$, and $\mathrm{Zn}$ which enhances their bio-activity ${ }^{10-11}$. 
International Journal of Current Science Research and Review

ISSN: 2581-8341

Volume 04 Issue 07 July 2021

DOI: 10.47191/ijcsrr/V4-i7-09, Impact Factor: 5.825

IJCSRR@ 2021

www.ijcsrr.org

Spectral data of 2-Amino-4H-1, 3-oxazine / thiazine:

\begin{tabular}{|c|c|c|c|c|c|c|}
\hline Sr.No & Name of Compound & Nature & M.P & $\begin{array}{l}\text { Result } \\
\text { in } \%\end{array}$ & H NMR & IR \\
\hline 1 & $\begin{array}{l}\text { 4,6-Dipheny1-4H-1,3- } \\
\text { Oxzine-2-amine }\end{array}$ & $\begin{array}{l}\text { pale } \\
\text { yellow } \\
\text { crystals }\end{array}$ & $\begin{array}{l}219- \\
221^{0} \mathrm{c}\end{array}$ & $89 \%$ & $\begin{array}{l}9.45\left(\mathrm{~S}, 2 \mathrm{H} . \mathrm{NH}_{2}\right) \\
7.76\left(\mathrm{~m}, \mathrm{~J}=2 \mathrm{~Hz}_{\mathrm{z}} 4 \mathrm{H}_{\mathrm{z}}, 2 \mathrm{H}, \mathrm{Ar}\right) \\
7.46(\mathrm{M}, 3 \mathrm{H}, \mathrm{HAr}) \\
7.52\left(\mathrm{~m}, \mathrm{~J}=2 \mathrm{~Hz}_{\mathrm{Z}}, 4 \mathrm{H}_{\mathrm{z}}, 8 \mathrm{H}, \mathrm{HAr}\right) \\
6.62 \quad\left(\mathrm{~d} \mathrm{~J}=4 \mathrm{H}_{\mathrm{z}}, 1 \mathrm{H}, \mathrm{CH}\right) \\
5.58\left(\mathrm{~d} \mathrm{~J}=4 \mathrm{H}_{\mathrm{z}}, 1 \mathrm{H}, \mathrm{CH}\right)\end{array}$ & $\begin{array}{lr}3400-3500, & 3078, \\
1710, & 1664, \\
1371, & 1330, \\
1263,1096 & \end{array}$ \\
\hline 2 & $\begin{array}{l}\text { 6-pheny-4 (P-toly) -4H- } \\
\text { 1-3-oxazine-2-amine }\end{array}$ & $\begin{array}{l}\text { pale } \\
\text { yellow } \\
\text { crystal }\end{array}$ & $\begin{array}{l}176- \\
178^{\circ} \mathrm{c}\end{array}$ & $84 \%$ & $\begin{array}{l}9.43(\mathrm{~S}, 2 \mathrm{H}, \mathrm{NH} 2) \\
7.72\left(\mathrm{~m} \mathrm{~J}=2 \mathrm{~Hz}, 4 \mathrm{H}_{\mathrm{Z}}, 2 \mathrm{H}, \mathrm{HAr}\right), 7.46 \\
(\mathrm{~m}, 3 \mathrm{H}, \mathrm{HAr}) \\
7.28\left(\mathrm{~d} \mathrm{~J}=4 \mathrm{H}_{\mathrm{z}}, 2 \mathrm{H}, \mathrm{Ar}\right) \\
7.16\left(\mathrm{~d}, \mathrm{~J}=4 \mathrm{H}_{\mathrm{Z}}, 2 \mathrm{H}, \mathrm{HAr}\right) \\
6.60\left(\mathrm{~d}, \mathrm{~J}=4 \mathrm{H}_{\mathrm{z}}, 1 \mathrm{H} \mathrm{CH}\right) \\
5.56\left(\mathrm{~d}, \mathrm{~J}=4 \mathrm{H}_{\mathrm{Z}}, 1 \mathrm{H} ; \mathrm{CH}\right) \\
2.43(\mathrm{~S}, 3 \mathrm{H}, \mathrm{CH} 3)\end{array}$ & 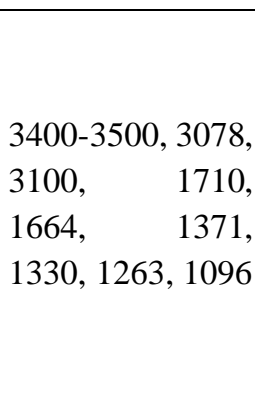 \\
\hline 3 & $\begin{array}{l}\text { 4-(O-Methoxyphenyl-6 } \\
\text {-phyenyl - 4H-1, 3- } \\
\text { Oxazine - 2-amine) }\end{array}$ & $\begin{array}{l}\text { Pale } \\
\text { yellow } \\
\text { crystal }\end{array}$ & $\begin{array}{l}186- \\
188^{\circ} \mathrm{c}\end{array}$ & $72 \%$ & $\begin{array}{l}9.42(\mathrm{Cs} 2 \mathrm{H}, \mathrm{NH} 2), \\
7.72\left(\mathrm{~m}, \mathrm{~J}=2 \mathrm{H}_{\mathrm{z}}, 4 \mathrm{H}_{\mathrm{z}}, 2 \mathrm{H}, \mathrm{HAr}\right) \\
7.43\left(\mathrm{~m}, \mathrm{~J}=2 \mathrm{H}_{\mathrm{Z}} 4 \mathrm{H}_{\mathrm{Z}}, 5 \mathrm{H}, \mathrm{HAr}\right), 7.22 \\
\left(\mathrm{~d}, \mathrm{~J}=4 \mathrm{~Hz}_{\mathrm{z}}\right. \\
7.43\left(\mathrm{~m}, \mathrm{~J}=2 \mathrm{~Hz}_{\mathrm{z}} 4 \mathrm{~Hz}_{\mathrm{z}}, 5 \mathrm{H}, \mathrm{HAr}\right), 7.22 \\
\left(\mathrm{~d}, \mathrm{~J}=4 \mathrm{H}_{\mathrm{Z}}, 1 \mathrm{H}, \mathrm{HAr}\right), \\
7.09\left(\mathrm{~d} \mathrm{~J}=4 \mathrm{H}_{\mathrm{Z}}, 1 \mathrm{H} \mathrm{HAr}\right), \\
6.61\left(\mathrm{~d}, \mathrm{~J}=4 \mathrm{H}_{\mathrm{Z}} 1 \mathrm{H} \mathrm{CH}\right), \\
5.54\left(\mathrm{~d}, \mathrm{~J}=4 \mathrm{H}_{\mathrm{Z}}, 1 \mathrm{H} \mathrm{CH}\right) \\
3.87\left(\mathrm{~S}, 3 \mathrm{H}, \mathrm{OCH}_{3}\right)\end{array}$ & 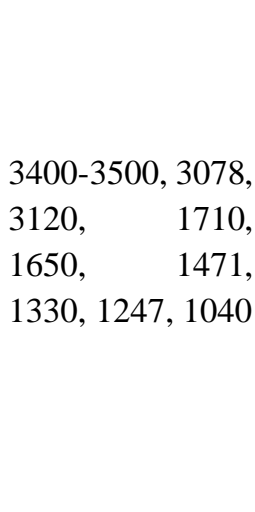 \\
\hline 4 & $\begin{array}{l}\text { (m-Nitrophenyl) } \\
\text { Pheuyl- } 4 \mathrm{H}-13 \text { oxazine- } \\
\text { 2-amine }\end{array}$ & $\begin{array}{l}\text { Pale } \\
\text { yellow } \\
\text { crystal }\end{array}$ & $\begin{array}{l}168- \\
170^{\circ} \mathrm{c}\end{array}$ & $86 \%$ & $\begin{array}{l}9.41(\mathrm{~s}, 2 \mathrm{H} \mathrm{NH} 2) \\
8.21\left(\mathrm{~m}, \mathrm{~J}=2 \mathrm{H}_{\mathrm{Z}}, 4 \mathrm{H}_{\mathrm{Z}}, 2 \mathrm{H}, \mathrm{HAr}\right) \\
7.99\left(\mathrm{~m}, \mathrm{~J}=2 \mathrm{H}_{\mathrm{Z}}, 4 \mathrm{H}_{\mathrm{Z}}, 2 \mathrm{H}, \mathrm{HAr}\right) \\
7.72(\mathrm{~m} .2 \mathrm{H}, \mathrm{HAr}) \\
6.42\left(\mathrm{~d}, \mathrm{~J}=4 \mathrm{H}_{\mathrm{z}} 1 \mathrm{H}, \mathrm{CH}\right) \\
5.55(\mathrm{~d}, \mathrm{~J}=4 \mathrm{H} 2,1 \mathrm{H}, \mathrm{CH})\end{array}$ & $\begin{array}{ll}3400-3500, & 3078, \\
1710, & 1664, \\
1371, & 1330, \\
1263,1096 & \end{array}$ \\
\hline 5 & $\begin{array}{l}\text { 4,6-diphenyl-4H-1, } 3 \text { - } \\
\text { Thiazine-2-amine }\end{array}$ & $\begin{array}{l}\text { Pale } \\
\text { yellow } \\
\text { crystals }\end{array}$ & $\begin{array}{l}178- \\
180^{\circ} \mathrm{c}\end{array}$ & $76 \%$ & $\begin{array}{l}9.88(\mathrm{~S} 2 \mathrm{H}, \mathrm{NH} 2) \\
7.42(\mathrm{M}, 7 \mathrm{H}, \mathrm{HAR}) \\
7.38(\mathrm{M}, 2 \mathrm{H}, \mathrm{HAr}) \\
7.18(\mathrm{~d} \mathrm{~J}=4 \mathrm{~Hz}, 1 \mathrm{H}, \mathrm{HAr}) \\
6.62\left(\mathrm{~d}, \mathrm{~J}=2 \mathrm{H}_{\mathrm{Z}} 1 \mathrm{H} \mathrm{CH}\right) \\
6.22\left(\mathrm{~d}, \mathrm{~J}=2 \mathrm{H}_{\mathrm{Z}} 1 \mathrm{H} \mathrm{CH}\right)\end{array}$ & $\begin{array}{ll}3400-3500, & 3080, \\
1523, & 1710, \\
1660,1371 & 1330, \\
1263,1096 & \end{array}$ \\
\hline
\end{tabular}


International Journal of Current Science Research and Review

ISSN: 2581-8341

Volume 04 Issue 07 July 2021

DOI: 10.47191/ijcsrr/V4-i7-09, Impact Factor: 5.825

www.ijcsrr.org

\begin{tabular}{|c|c|c|c|c|c|c|}
\hline 6 & $\begin{array}{l}6 \quad \text { (phenyl-4(P-Tolyl)- } \\
4 \mathrm{H}-1, \quad 3 \text { thiazin- 2- } \\
\text { amine }\end{array}$ & $\begin{array}{l}\text { Pale } \\
\text { yellow } \\
\text { crystal }\end{array}$ & $\begin{array}{l}180- \\
182^{\circ} \mathrm{c}\end{array}$ & $72 \%$ & $\begin{array}{l}9.86(\mathrm{~S}, 2 \mathrm{H}, \mathrm{NH} 2) \\
7.41\left(\mathrm{~d}, \mathrm{~J}=8 \mathrm{~Hz}_{\mathrm{z}}, 1 \mathrm{H}, \mathrm{HAR}\right) \\
7.34\left(\mathrm{~d}, \mathrm{~J}=8 \mathrm{H}_{\mathrm{Z}}, 1 \mathrm{H}, \mathrm{HAR}\right), \\
7.22(\mathrm{~m}, 7 \mathrm{H} \mathrm{HAr}), \\
6.71\left(\mathrm{~d}, \mathrm{~J}=2 \mathrm{H}_{\mathrm{Z}}, 1 \mathrm{H}, \mathrm{CH}\right), \\
6.24\left(\mathrm{~d}, \mathrm{~J}=2 \mathrm{H}_{\mathrm{z}}, 1 \mathrm{H}, \mathrm{CH}\right) \\
2.42(\mathrm{~S}, 3 \mathrm{H}, \mathrm{CH} 3)\end{array}$ & 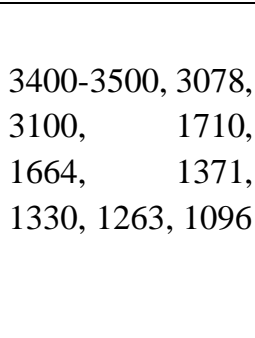 \\
\hline 7 & $\begin{array}{l}4 \text { (P-Chlorophenyl) } \\
\text { phenyl-4 H-1, } \\
\text { thiazine-2amine }\end{array}$ & $\begin{array}{l}\text { Pale } \\
\text { yellow } \\
\text { crystals }\end{array}$ & $\begin{array}{l}170- \\
172^{\circ} \mathrm{c}\end{array}$ & $77 \%$ & $\begin{array}{l}9.88(\mathrm{~S}, 2 \mathrm{H}, \mathrm{NH} 2) \\
7.42(\mathrm{~m}, 4 \mathrm{~Hz}, \mathrm{HAr}), \\
7.32(\mathrm{~m}, 2 \mathrm{H}, \mathrm{HAr}) \\
6.26(\mathrm{M}, 3 \mathrm{H}, \mathrm{HAr}) \\
6.72\left(\mathrm{~d}, \mathrm{~J}=2 \mathrm{~Hz}_{\mathrm{z}} 1 \mathrm{H} \mathrm{CH}\right), \\
6.40\left(\mathrm{~d}, \mathrm{I}=2 \mathrm{H}_{\mathrm{z}}, 1 \mathrm{H}, \mathrm{CH}\right)\end{array}$ & $\begin{array}{ll}3400-3500, & 3078, \\
1720, & 1660, \\
1371, & 1330, \\
1263,1096 & \end{array}$ \\
\hline
\end{tabular}

Spectral data of Schiff's bases

\begin{tabular}{|c|c|c|c|}
\hline $\begin{array}{l}\text { Sr. } \\
\text { No }\end{array}$ & Name of Compound & H NMR & IR \\
\hline 1 & $\begin{array}{l}4,6 \quad \text { Dipheny-4H-1, } 3 \\
\text { Oxazine-2, imine } \\
\text { hydroxyl) benzene }\end{array}$ & $\begin{array}{l}7.76\left(\mathrm{~m}, \mathrm{~J}=2 \mathrm{~Hz}_{\mathrm{z}}, 4 \mathrm{H}_{\mathrm{z}}, 2 \mathrm{H}, \mathrm{Ar}\right), 7.52(\mathrm{~m}, \\
\left.\mathrm{J}=2 \mathrm{H}_{\mathrm{z}}, 4 \mathrm{H}_{\mathrm{z}}, 8 \mathrm{H}, \mathrm{HAr}\right) \\
6.62\left(\mathrm{t}, \mathrm{J}=4 \mathrm{H}_{\mathrm{z}}, 1 \mathrm{H} \mathrm{CH}\right) \\
5.58\left(\mathrm{~d}, \mathrm{~J}=4 \mathrm{H}_{\mathrm{z}}, 1 \mathrm{H} \mathrm{CH}\right) \\
5.48\left(\mathrm{~d} \mathrm{~J}=2 \mathrm{H}_{\mathrm{Z}} 1 \mathrm{H} \mathrm{CH}\right) \\
5.20(\mathrm{~S} 1 \mathrm{H}, \mathrm{OH})\end{array}$ & $\begin{array}{l}3650-3584,3080,1710,1664,1470,1263, \\
1096\end{array}$ \\
\hline 2 & $\begin{array}{l}\text { 6- Phenyl -4 ( P-tolyl)- 4H-1-3 } \\
\text { Oxazine - } 2 \text { imine (2-hydroxy) } \\
\text { benzene }\end{array}$ & $\begin{array}{l}7.72\left(\mathrm{~m}, \mathrm{~J}=2 \mathrm{H}_{\mathrm{Z}} 4 \mathrm{H}_{\mathrm{z}}, 2 \mathrm{H}, \mathrm{Ar}\right) 7.46(\mathrm{~m}, 3 \mathrm{H} \\
\mathrm{Har}) \\
7.28\left(\mathrm{~d} \mathrm{~J}=4 \mathrm{H}_{\mathrm{z}} 2 \mathrm{H}, \mathrm{HAr}\right) \\
7.16\left(\mathrm{~d}, \mathrm{~J}=4 \mathrm{H}_{\mathrm{z}}, 2 \mathrm{H}, \mathrm{HAr}\right) \\
6.62\left(\mathrm{~d} \mathrm{~J}=4 \mathrm{H}_{\mathrm{z}}, 1 \mathrm{H} \mathrm{CH}\right) \\
5.56\left(\mathrm{t}, \mathrm{J}=4 \mathrm{H}_{\mathrm{z}} 1 \mathrm{H}, \mathrm{CH}\right) \\
5.48\left(\mathrm{~d}, 2 \mathrm{H}_{\mathrm{Z}}, 1 \mathrm{H}, \mathrm{CH}\right) \\
5.20(\mathrm{~S}, 1 \mathrm{H}, \mathrm{OH}) \\
2.43(\mathrm{~S}, 3 \mathrm{H}, \mathrm{CH} 3)\end{array}$ & $\begin{array}{l}3650-3584,3080,1710,1689,1650,1470, \\
1263,1096\end{array}$ \\
\hline 3 & $\begin{array}{l}\text { 4- }(\mathrm{O}-\text { methoxy phenyl }-6 \\
\text { phenyl - 4H,1 3-Oxazine 2- } \\
\text { Imine (2-hydroxy) benzene }\end{array}$ & $\begin{array}{l}7.72\left(\mathrm{~m}, \mathrm{~J}-2 \mathrm{H}_{\mathrm{z}}, 4 \mathrm{H}_{\mathrm{z}}, 2 \mathrm{H} \mathrm{HAr}\right), 7.43 \\
\left(\mathrm{~m}, \mathrm{~J}=2 \mathrm{H}_{\mathrm{Z}} 4 \mathrm{H}_{\mathrm{z}}, 5 \mathrm{H} \mathrm{HAr}\right), \\
7.22\left(\mathrm{~d}, \mathrm{~J}=4 \mathrm{H}_{\mathrm{z}}, 1 \mathrm{H}, \mathrm{HAr}\right) \\
7.09\left(\mathrm{~d} \mathrm{~J}=4 \mathrm{H}_{\mathrm{z}}, 1 \mathrm{H}, \mathrm{HAr}\right) \\
6.61\left(\mathrm{~d} \mathrm{~J}=4 \mathrm{H}_{\mathrm{z}}, 1 \mathrm{H} \mathrm{CH}\right) \\
5.54\left(\mathrm{~d} \mathrm{~J}=4 \mathrm{H}_{\mathrm{z}} 1 \mathrm{H} \mathrm{CH}\right) \\
5.48(\mathrm{~d} 2 \mathrm{H} 1 \mathrm{H} \mathrm{CH}) \\
5.20(\mathrm{~S}, 1 \mathrm{H}, \mathrm{OH}) \\
3.87(\mathrm{~S}, 3 \mathrm{H}, \mathrm{OCH} 3)\end{array}$ & $\begin{array}{l}3650-3584,3080,3120,1710,1630,1471, \\
1330,1247,1040\end{array}$ \\
\hline
\end{tabular}


ISSN: 2581-8341

Volume 04 Issue 07 July 2021

DOI: 10.47191/ijesrr/V4-i7-09, Impact Factor: 5.825

\begin{tabular}{|c|c|c|c|}
\hline 4 & $\begin{array}{l}\text { (M-Nitrophyenyl) } 1-6 \\
\text { Phyenyl-4H-1, 3- Pxazome-2 } \\
\text { imine (2-hydroxy)benzene }\end{array}$ & $\begin{array}{l}8.21(\mathrm{~m}, \mathrm{~J}=2 \mathrm{~Hz}, 4 \mathrm{~Hz}, 2 \mathrm{H}, \mathrm{HAr}), 7.99(\mathrm{~m}, \\
\mathrm{J}=2 \mathrm{z}, 4 \mathrm{z}, 2 \mathrm{H}, \mathrm{HAr}) \\
7.72(\mathrm{M}, 2 \mathrm{H}, \mathrm{HAr}) \\
6.42(\mathrm{D}, \mathrm{J}=4 \mathrm{z}, 1 \mathrm{H}, \mathrm{CH}) \\
5.55(\mathrm{~d}, \mathrm{~J}-4 \mathrm{~Hz}, 1 \mathrm{H}, \mathrm{CH}) \\
5.48(\mathrm{~d}, \mathrm{~J}=2 \mathrm{~Hz}, 1 \mathrm{H} \mathrm{CH}) \\
5.20(\mathrm{~S}, 1 \mathrm{H}, \mathrm{OH})\end{array}$ & $\begin{array}{l}3650-3584,3080,1710,1630,1471,1330, \\
1247,1040\end{array}$ \\
\hline 5 & $\begin{array}{l}4, \quad 6 \text { diphenyl-4H-1, } 3- \\
\text { thiazine-2 imine (2-Hydroxy) } \\
\text { benzene }\end{array}$ & $\begin{array}{l}7.42(\mathrm{~m}, 7 \mathrm{H}, \mathrm{HAr}) \\
7.38(\mathrm{~m}, 2 \mathrm{H}, \mathrm{HAr}) \\
7.18(\mathrm{~d}, \mathrm{~J}=4 \mathrm{~Hz}, 1 \mathrm{H}, \mathrm{HAr}) \\
6.62(\mathrm{~d}, \mathrm{~J}=2 \mathrm{~Hz}, 1 \mathrm{H}, \mathrm{CH}) \\
6.22(\mathrm{~d}, \mathrm{~J}=2 \mathrm{z}, 1 \mathrm{H}, \mathrm{CH}) \\
5.58(\mathrm{~d}, \mathrm{~J}=2 \mathrm{~Hz}, 1 \mathrm{H}, \mathrm{CH}) \\
5.20(\mathrm{~S}, 1 \mathrm{H}, \mathrm{OH})\end{array}$ & $\begin{array}{l}3650-3584,3080,1710,1660,1371,1330, \\
1263,1096\end{array}$ \\
\hline 6 & $\begin{array}{l}\text { 6-Phenyal-4 (P-tolyl)-4H-1, } 3 \\
\text {-Thizine-2-imine } \\
\text { hydroxy)benzene }\end{array}$ & $\begin{array}{l}7.41(\mathrm{~d}, \mathrm{~J}=8 \mathrm{~Hz}, 1 \mathrm{H}, \mathrm{HAr}) \\
7.34(\mathrm{~d}, \mathrm{~J}=8 \mathrm{~Hz}, 1 \mathrm{H} \mathrm{HAr}) \\
7.22(\mathrm{~m}, 7 \mathrm{H}, \mathrm{HAr}) \\
6.71(\mathrm{~d}, \mathrm{~J}=2 \mathrm{~Hz}, \mathrm{CH}) \\
6.24(\mathrm{~d}, \mathrm{~J}=2 \mathrm{~Hz}, 1 \mathrm{H}, \mathrm{CH}) \\
5.58(\mathrm{~d} \mathrm{~J}=2 \mathrm{~Hz}, 1 \mathrm{H}, \mathrm{CH}) \\
5.20(\mathrm{~S}, 1 \mathrm{H}, \mathrm{OH}) \\
2.42(\mathrm{~S}, 3 \mathrm{H}, \mathrm{CH} 3)\end{array}$ & $\begin{array}{l}3650-3584,3078,3100,1710,1664,1371, \\
1330,1263,1096\end{array}$ \\
\hline 7 & $\begin{array}{l}4 \text { (P.Chlorophenyl) -6 phenyl- } \\
4 \text { H-1, 3-thiazine- 2-imine (2- } \\
\text { hydroxy) benzene }\end{array}$ & $\begin{array}{l}7.42(\mathrm{~m}, 4 \mathrm{H}, \mathrm{HAr}) \\
7.32(\mathrm{~m}, 2 \mathrm{H}, \mathrm{HAr}) \\
7.26(\mathrm{~m}, 3 \mathrm{H}, \mathrm{HAr}) \\
6.72\left(\mathrm{~d}, \mathrm{~J}=2 \mathrm{H}_{\mathrm{Z}} 1 \mathrm{H} \mathrm{CH}\right) \\
6.60(\mathrm{~d}, \mathrm{~J}=2 \mathrm{H} 21 \mathrm{H} \mathrm{CH}) \\
5.50(\mathrm{~d}, \mathrm{I}=2 \mathrm{~Hz}, 1 \mathrm{H}, \mathrm{CH}) \\
5.20(\mathrm{~S}, \mathrm{~J} 1 \mathrm{H} \mathrm{OH})\end{array}$ & $\begin{array}{l}3650-3584,3078,1710,1660,1371,1330, \\
1263,1096\end{array}$ \\
\hline 8 & $\begin{array}{l}4 \text { (P-Nitrophyanyl ) -6 phenyl- } \\
4 \text { H-1, 3-thiazine- 2-imine (2- } \\
\text { hydroxy)benzene }\end{array}$ & $\begin{array}{l}8.32(\mathrm{~m}, 2 \mathrm{H}, \mathrm{HAr}) \\
7.82(\mathrm{~m}, 2 \mathrm{H}, \mathrm{HAr}) \\
7.45(\mathrm{~m}, 5 \mathrm{H}, \mathrm{HAr}) \\
6.67(\mathrm{~d}, \mathrm{~J}=2 \mathrm{~Hz}, 1 \mathrm{H} \mathrm{CH}) \\
6.26(\mathrm{~d}, \mathrm{~J}=2 \mathrm{H} 2,1 \mathrm{H} \mathrm{CH}), \\
5.58(\mathrm{~d}, \mathrm{~J}=2 \mathrm{H} 21 \mathrm{H} \mathrm{CH}), \\
5.20(\mathrm{~S}, 1 \mathrm{H} \mathrm{CH})\end{array}$ & $\begin{array}{l}3650-3584,3078,1710,1660,1523,1371, \\
1330,1263,1096\end{array}$ \\
\hline
\end{tabular}

\section{CONCLUSION}

All the synthesized Schiff' Bases and their metal complexes having bioactive properties like anti-inflammatory, antitumor, antibiotic, antifungal activities.

\section{REFERENCES}

1. Mathew, B.P. Kumar, A.Sharma, S. Shukla, P.K. Nath, M.Eur, J. Med. Chem. 45, 1502 (2010)

2. Haneisi J. : Okazaki, T. Hata, T, Jamura L, Nom. Arura M.J. Antibiot,24, 797 (1971)

3. Sasaki K. Kusalabe, Y. Esumi, S.J. Aautibiot 25,151(1972)

4. Pictsh M : Custchow M.J. Med. Chem. 48,8270 (2005) 


\section{International Journal of Current Science Research and Review}

ISSN: 2581-8341

Volume 04 Issue 07 July 2021

DOI: 10.47191/ijcsrr/V4-i7-09, Impact Factor: 5.825

IJCSRR@ 2021

www.ijjcsrr.org

5. Hung S. : Pan Y. : Zhu Y : Wu, A. Org, Lett. 7,3797 (2005)

6. Cecchetti, V : Cruciani, G : Filliponi , E : Fravolini A; Tabarrini O, T.Bioorg. Med.Chem. 5, 1339(1997)

7. Remillard, S. Rebhu, L.I Howie, G.A. Kupchan, S.M .Science 189 ,1002 (1975)

8. Sokolova, A,S ; Rayabokon', N.A; Erashova, Yu.A; Andreeva, N.A. ; Nemeryuk, M.P; Kermov, A.F. Traven, N.I; Yadrovskaya, V.A; Chernov, V.A ; Safonova, T.S.Pharm. Chem.J 11 (9) ,49 (1977)

9. Pietsch, M, Gutschow M, M.J.Med.Chem, 48,8270 (2005)

10. Diwagh, S.S; Piste, P.B. Int. J. Pharm. Sci, Res. 4,2045 (2013)

11. Mandal, P.K.Misra, A.K. Lett. Org. Chem. 3, 848 (2006)

Cite this Article: D.T. Biradar, Dr. M. B. Swami (2021). Synthesis of Schiff's Bases from 2-Amino-4H-1, 3-Oxazine /Thiazine and Substituted Aldehydes and their Transition Metal Complexes. International Journal of Current Science Research and Review, 4(7), 677-683 